\title{
Homeomorphisms of inverse limit spaces of one-dimensional maps
}

\author{
by \\ Marcy B a r ge (Bozeman, Mt.) and \\ Beverly Diamond (Charleston, S.C.)
}

\begin{abstract}
We present a new technique for showing that inverse limit spaces of certain one-dimensional Markov maps are not homeomorphic. In particular, the inverse limit spaces for the three maps from the tent family having periodic kneading sequence of length five are not homeomorphic.
\end{abstract}

1. Introduction. Inverse limit spaces of one-dimensional maps commonly appear as attractors of dynamical systems (see, for example, [2], [3], [12], [19]). It is then of interest to determine when such inverse limit spaces are homeomorphic. In general, this appears to be a difficult question.

Let $f_{n}$ denote the map of the unit interval $I$ with $f_{n}(m / n)=0$ if $m$ is even and $f_{n}(m / n)=1$ if $m$ is odd $(m=0,1, \ldots, n)$, and which is linear in between these points. Let $\left(I, f_{n}\right)$ denote the inverse limit space obtained by using $f_{n}$ as a single bonding map. By investigating the combinatorial structure of the composants containing the fixed point $(0,0,0, \ldots)$, Watkins [18] proved that if $n$ and $m$ do not have the same prime factors, then $\left(I, f_{n}\right)$ and $\left(I, f_{m}\right)$ are not homeomorphic. Dębski $([7])$ has generalized this to cover inverse limits constructed using multiple bonding maps selected from the $f_{n}$. (The corresponding classification problem for solenoids has also been solved; see [5], [14] and [1].)

Define the family of tent maps $f_{\lambda}: I \rightarrow I$, for $0 \leq \lambda \leq 2$, as

$$
f_{\lambda}(x)= \begin{cases}\lambda x, & 0 \leq x \leq .5 \\ \lambda(1-x), & .5 \leq x \leq 1\end{cases}
$$

In terms of the topology of the inverse limit space, the map $f_{\lambda}$ serves as a prototype for certain unimodal maps having the same kneading sequence. For

1991 Mathematics Subject Classification: Primary 54F15; Secondary 54H20, 58F03, $58 \mathrm{~F} 12$. 
example, if the kneading sequence of $f_{\lambda}$ is finite, and $g$ is any unimodal map with identical kneading sequence, the inverse limit space $(I, g)$ is homeomorphic to $\left(I, f_{\lambda}\right)$ ([11], see also [9] for results of this sort). At the Spring Topology Conference in 1992, T. Ingram asked whether members of the tent family having distinct periodic kneading sequences of the same length can have homeomorphic inverse limit spaces. (Unpublished work by Barge and Martin indicates that for maps having periodic kneading sequences of different lengths, the inverse limit spaces are distinct in virtue of having a different number of endpoints.) While the inverse limit space of a map in the tent family having periodic kneading sequence can be represented symbolically, the combinatorial structure of the composants is sufficiently complicated so as to make the generalization of Watkins' techniques difficult.

In this paper, we present another technique for showing that inverse limit spaces of one-dimensional maps are not homeomorphic. We prove that for transitive Markov maps $f$ and $g$ of the interval, the existence of a homeomorphism between inverse limit spaces leads to a commuting diagram of "transition" matrices (Theorem 3.4) and a relationship between the eigenvalues of the transition matrices for $f$ and $g$ (Corollary 3.5). Combining this with work of Lind [13], we deduce that if $(I, f)$ and $(I, g)$ are homeomorphic, the algebraic extensions $\mathbb{Q}(\alpha)$ and $\mathbb{Q}(\beta)$ are equal, where $\alpha$ and $\beta$ are the largest (in absolute value) and real eigenvalues (i.e., the spectral radii) of the transition matrices for $f$ and $g$ (Corollary 3.5). Applying this to the three maps from the tent family with periodic kneading sequence of length five, we conclude that the inverse limit spaces for all three are distinct (Theorem 4.2), answering Ingram's question in this case.

The outline of the paper is as follows. In $\S 2$, we introduce notation and mention some known results. In $\S 3$, we state the main results and prove all except Theorem 3.4; the details of Theorem 3.4 are somewhat technical and appear in $\S 5$. In $\S 4$, we apply the main results to the case of the three period fives.

2. Notation and preliminaries. Given a continuous function $f: I \rightarrow I$ of the interval $I=[0,1]$, the associated inverse limit space $(I, f)$ with single bonding map $f$ is defined by

$$
(I, f)=\left\{\underline{x}=\left(x_{0}, x_{1}, \ldots\right): x_{n} \in I, f\left(x_{n+1}\right)=x_{n}, n=0,1, \ldots\right\} .
$$

A continuous map $f: I \rightarrow I$ is Markov if there is a finite collection of points $0=c_{0}<c_{1}<\ldots<c_{n}=1$ such that $f\left(\left\{c_{0}, c_{1}, \ldots, c_{n}\right\}\right) \subseteq\left\{c_{0}, c_{1}, \ldots, c_{n}\right\}, f$ is not constant on $\left[c_{i-1}, c_{i}\right]$ for any $i$, and each interval $\left[c_{i-1}, c_{i}\right], i=1, \ldots, n$, is a finite union of subintervals $[a, b]$ such that $\left.f\right|_{[a, b]}$ is monotone and $f(\{a, b\}) \subseteq\left\{c_{0}, c_{1}, \ldots, c_{n}\right\}$. The collection $\left\{c_{0}, c_{1}, \ldots, c_{n}\right\}$ will be called a 
Markov partition for $f$. If $f$ is Markov with Markov partition $\left\{c_{0}, c_{1}, \ldots, c_{n}\right\}$, the transition matrix of $f$ with respect to $\left\{c_{0}, c_{1}, \ldots, c_{n}\right\}$ is the $n \times n$ integer matrix $C=\left(c_{i j}\right)$ where $c_{i j}$ is the number of times $\left.f\right|_{\left[c_{j-1}, c_{j}\right]}$ covers $\left[c_{i-1}, c_{i}\right]$ (so $c_{i j}$ is the number of components of $\left(\left.f\right|_{\left[c_{j-1}, c_{j}\right]}\right)^{-1}\left(\left(c_{i-1}, c_{i}\right)\right)$ and each component maps onto $\left.\left(c_{i-1}, c_{i}\right)\right)$. The matrix $C$ is aperiodic or eventually positive if there is a positive integer $m$ such that every entry of $C^{m}$ is positive. For technical reasons, we say that a Markov map $f$ is aperiodic if its transition matrix $C$ is aperiodic and either $n \geq 2$ or $n=1$ and $c_{11}>1$.

If $f$ is Markov, there is a second Markov map $g$ with the same Markov partition, the same transition matrix and no flat spots (that is, $g$ is nowhere locally constant) such that the inverse limit space $(I, f)$ of $I$ with single bonding map $f$ is homeomorphic to $(I, g)$ ([10, Theorem 3.2]). Suppose that $f$ is Markov with Markov partition $\left\{c_{0}, c_{1}, \ldots, c_{n}\right\}$ and that $f$ has no flat spots. A point $c \in I$ is a critical point of $f$ if either $c \in\{0,1\}$ or $\left.f\right|_{J}$ is not one-to-one for each subinterval $J$ with $c \in \operatorname{int}(J)$. If $c \in(0,1)$ is a critical point of $f$, we will call $f(c)$ a critical value of $f$. Suppose that $f$ is onto, so that the critical values of $f^{k+1}$ contain the critical values of $f^{k}$ for each $k \geq 1$. Since the collection of critical values of $f$ is contained in $\left\{c_{0}, c_{1}, \ldots, c_{n}\right\}$ and $f\left(\left\{c_{0}, c_{1}, \ldots, c_{n}\right\}\right) \subseteq\left\{c_{0}, c_{1}, \ldots, c_{n}\right\}$, there is an $l>0$ such that the critical values of $f^{l}$ are the same as the critical values of $f^{k}$ for all $k \geq l$. If $\mathcal{C}^{\prime}$ is the collection of critical values of $f^{l}$ and $\mathcal{C}^{\prime} \cup$ $\{0,1\}=\left\{0=c_{0}^{\prime}, c_{1}^{\prime}, \ldots, c_{m}^{\prime}=1\right\}\left(\subseteq\left\{c_{0}, c_{1}, \ldots, c_{n}\right\}\right)$, then $\left\{c_{0}^{\prime}, c_{1}^{\prime}, \ldots, c_{m}^{\prime}\right\}$ is a Markov partition for $f^{l}$ with the property that for $0<i<m, c_{i}^{\prime}$ is a critical value of $f^{l}$. If $f$ is aperiodic, we may choose $l$ large enough so that 0 and 1 are also critical values, each element of the partition is actually a critical point, and each critical value is realized at least $k$ times for a fixed positive integer $k$. (The last assumption provides a critical point in the interior of $I$ realizing a particular critical value for $k \geq 3$.) Since $\left(I, f^{l}\right)$ is homeomorphic to $(I, f)$, we have established (a) through (e) of the following.

LEMMA 2.1. If $f$ is aperiodic, there is an aperiodic (Markov) function $g$ with the following properties:

(a) g has no flat spots,

(b) the Markov partition $\mathcal{C}$ for $g$ equals the set of critical values for $g$ and is contained in the set of critical points for $g$,

(c) each critical value is realized by at least three critical points of $I$,

(d) $g[c, d]=I$ for distinct $c, d \in \mathcal{C}$,

(e) $(I, g)$ is homeomorphic to $(I, f)$,

(f) $g$ is piecewise linear with slope everywhere equal in absolute value to the spectral radius of the transition matrix for $g$. 
Proof. According to the preceding remarks, we need only show that (f) can be met. Suppose then that $f$ itself satisfies (a), (b), (c) and (d). Replace the Markov partition $\mathcal{C}$ for $f$ witnessing (b) and (d) by a finer Markov partition $P(f)$ for which each entry in the transition matrix is either a 0 or a 1 . Let $P(f)=\left\{0=a_{0}<a_{1}<\ldots<a_{n(f)}=1\right\}$ and $\left\{0=b_{0}<b_{1}<\ldots<b_{n(f)}=1\right\} \subseteq I$. Let $\phi: I \rightarrow I$ denote the homeomorphism with $\phi\left(a_{i}\right)=b_{i}$ for $0 \leq i \leq n(f)$ and which is linear in between these points. Then the map $g=\phi \circ f \circ \phi^{-1}$ is conjugate to $f$ on $I$, satisfies (a), (c) and (e) and has the same transition matrix as $f$. Since $g$ is strictly increasing or strictly decreasing between partition points, $g$ may be assumed to be linear between these points by $[10,3.2]$. If the partition $\left\{b_{0}, b_{1}, \ldots, b_{n(f)}\right\}$ is chosen so that for each $i, b_{i}-b_{i-1}$ is the $i$ th entry in the normalized left eigenvector corresponding to the spectral radius of the transition matrix, then (f) holds. Finally, there is a Markov partition for $g$ corresponding to the original Markov partition for $f$ (and thus with identical transition matrix) with the desired properties.

Note that the spectral radius is greater than one in the above result. Since the transition matrix is aperiodic, a subinterval of $I$ having nonempty interior eventually maps under $g$ over the entire interval $I$.

The Perron-Frobenius Theorem states that if $M$ is a nonnegative aperiodic matrix, then $M$ has a real positive eigenvalue which is larger than any other eigenvalue of $M$ in absolute value (and thus equals the spectral radius of $M$ ) and which is unique in having an eigenvector with all components positive ([8]). As in [13], we define the set $\mathcal{P}$ of Perron numbers to be the set of algebraic integers greater than 1 which have the property that they are larger than the modulus of all their algebraic conjugates. Lind has shown that any Perron number is realized as the spectral radius of an aperiodic nonnegative integral matrix ([13, Theorem 1]), thus the Perron numbers are precisely the spectral radii of such matrices. He also proves that $\mathcal{P}$ is closed under addition and multiplication ([13, Proposition 1]), and that if $\alpha \beta=\gamma$, where $\alpha, \beta, \gamma \in \mathcal{P}$, then $\alpha, \beta \in \mathbb{Q}(\gamma)$, the algebraic extension of the rationals $\mathbb{Q}$ by $\gamma([13$, Proposition 5$])$.

3. The main results. In the following, $f$ and $g$ are Markov maps of the interval with Markov partitions $P(f)=\left\{0=a_{0}<a_{1}<\ldots<a_{n(f)}=1\right\}$ and $P(g)=\left\{0=b_{0}<b_{1}<\ldots<b_{n(g)}=1\right\}$. We assume that both $f$ and $g$ satisfy the conclusions of Lemma 2.1.

The following result concerning the topology of the inverse limit space will be helpful. As usual, $\pi_{k}:(I, f) \rightarrow I$ and $\pi_{k}:(I, g) \rightarrow I$ denote projection onto the $k$ th coordinate, $k=1,2, \ldots$ 
Lemma 3.1. Suppose that $g$ is as above, and that $H$ is a proper subcontinuum of $(I, g)$. There is a positive integer $K$ so that $\left.\pi_{k}\right|_{H}$ is one-to-one for $k \geq K$.

Proof. Since $H$ is a proper subcontinuum of $(I, g)$, there is a positive integer $N$ so that for $n \geq N, \pi_{n}(H) \neq I$, and thus for $n \geq N+1, \pi_{n}(H)$ contains at most one critical value of $g$. If there exists $M$ such that for $m \geq M, \pi_{m}(H)$ does not contain any critical value of $H$, then $\pi_{m}$ is oneto-one on $H$ for $m \geq M$. If $\pi_{n}(H)$ contains a critical value for infinitely many $n \in \mathbb{Z}^{+}$, then there is a unique $\underline{x} \in(I, g)$ such that $x_{n} \in P(g)$ for infinitely many $n$ and thus for all $n \in \mathbb{Z}^{+}$. Suppose that for some $j>N+1$, $x_{j} \in \operatorname{int}\left(\pi_{j}(H)\right)$. Then $x_{j-1}$ is an endpoint of $\pi_{j-1}(H)$ since $g$ turns at $x_{j}$ and at no other point of $\pi_{j}(H)$. In fact, $x_{n}$ is an endpoint of $\pi_{n}(H)$ for $N+1 \leq n \leq j$. But then $x_{m} \notin \operatorname{int}\left(\pi_{m}(H)\right)$ for $m>j$, hence $\pi_{m}$ is one-to-one on $H$ for $m>j$.

Given a homeomorphism between $(I, f)$ and $(I, g)$ and a sequence $\left\{\varepsilon_{l}\right\}_{l \in \mathbb{Z}^{+}}$of positive numbers, Mioduszewski ([15]) constructs an infinite diagram of maps that $\varepsilon_{l}$-commute:
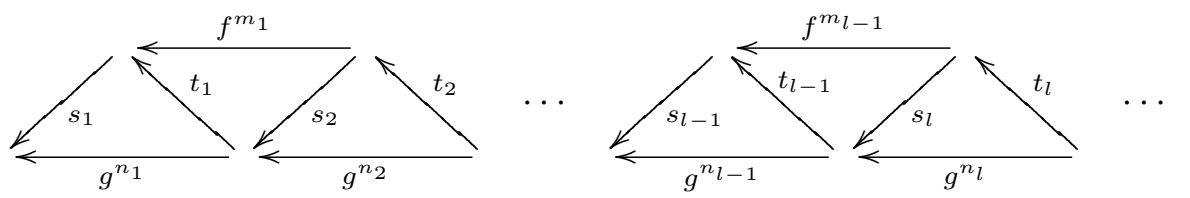

We wish to use such a diagram to obtain a diagram of commuting matrices (Theorem 3.4), and thus need to explicitly construct connecting maps which "almost" map $P(f)$ to $P(g)$ and $P(g)$ to $P(f)$. In the case in which, for each of $f$ and $g$, there is a subinterval mapping in a oneto-one fashion over the entire interval $I$, we are able to define a single connecting map in each direction at each stage of the construction. For technical reasons, when this is not the case, our techniques require several connecting maps defined on subintervals of $I$ at each stage of the diagram.

Proposition 3.2. Suppose that $(I, f)$ and $(I, g)$ are homeomorphic. Given a sequence $\left\{\varepsilon_{l}\right\}_{l \in \mathbb{Z}^{+}}$of positive numbers, there are sequences of positive integers $\left\{m_{l}\right\}_{l \in \mathbb{Z}^{+}}$and $\left\{n_{l}\right\}_{l \in \mathbb{Z}^{+}}$; points $\alpha_{i}^{-}, \alpha_{i}^{+} \in P(f)$ and $\beta_{j}^{-}, \beta_{j}^{+} \in$ $P(g)$ with $\alpha_{i}^{-} \leq a_{i-1}, \alpha_{i}^{+} \geq a_{i}, \beta_{j}^{-} \leq b_{j-1}, \beta_{j}^{+} \geq b_{j}$ for $1 \leq i \leq n(f)$ and $1 \leq j \leq n(g) ;$ and maps $s_{l, i}:\left[\alpha_{i}^{-}, \alpha_{i}^{+}\right] \rightarrow I, t_{l, j}:\left[\beta_{j}^{-}, \beta_{j}^{+}\right] \rightarrow I$ for $l \in \mathbb{Z}^{+}$, $1 \leq i \leq n(f)$ and $1 \leq j \leq n(g)$ such that:

(a) $\left|s_{l, i} \circ t_{l, j}-g^{n_{l}}\right|<\varepsilon_{l}$ and $\left|t_{l, j} \circ s_{l+1, i}-f^{m_{l}}\right|<\varepsilon_{l}$, where defined;

(b) $s_{l, i}$ and $t_{l, j}$ are piecewise monotone with no flat spots; 
(c) the critical values of $s_{l, i}$ (respectively, $t_{l, j}$ ) lie in $P(g)$ (respectively, $P(f)$ ); moreover, each element of $P(g)$ (respectively, $P(f)$ ) is a critical value of each $s_{l, i}$ (respectively, $t_{l, j}$ ) and is realized as such at least three times,

(d) $\alpha_{i}^{-}$(respectively, $\beta_{j}^{-}$) is a local minimum of each $t_{l, j}$ (respectively, $s_{l, i}$ ), and $\alpha_{i}^{+}$(respectively, $\beta_{j}^{+}$) is a local maximum of each $t_{l, j}$ (respectively, $\left.s_{l, i}\right)$.

Proof. We first prove the theorem under the assumption that $f$ and $g$ have right inverses $f_{1}: I \rightarrow L$ and $g_{1}: I \rightarrow L^{\prime}$ (that is, there exist subintervals $L$ and $L^{\prime}$ of $I$ and maps $f_{1}$ and $g_{1}$ onto $L$ and $L^{\prime}$ respectively such that $f \circ f_{1}: I \rightarrow I$ and $g \circ g_{1}: I \rightarrow I$ equal the identity on $\left.I\right)$. We then indicate the proof of the more general result. Define right inverses, $\hat{\imath}_{k}: I \rightarrow(I, f)$ and $\hat{\imath}_{k}: I \rightarrow(I, g)$, of the projection functions $\pi_{k}$ as follows:

$$
\hat{\imath}_{k}(x)=\left(f^{k}(x), f^{k-1}(x), \ldots, x, f_{1}(x), f_{1}^{2}(x), \ldots\right)
$$

or

$$
\hat{\imath}_{k}(x)=\left(g^{k}(x), g^{k-1}(x), \ldots, x, g_{1}(x), g_{1}^{2}(x), \ldots\right) .
$$

Suppose that $\Phi:(I, f) \rightarrow(I, g)$ is a homeomorphism. For $n, m \geq 0$, define $s(n, m), t(n, m): I \rightarrow I$ as

$$
s(n, m)=\pi_{m} \circ \Phi \circ \hat{\imath}_{n}, \quad t(n, m)=\pi_{m} \circ \Phi^{-1} \circ \hat{\imath}_{n} .
$$

Note that $g^{k} \circ s(n, m+k)=s(n, m)$ and $f^{k} \circ t(n, m+k)=t(n, m)$ for all $k \geq 0$. Fix $n, m \geq 0$. The map $\Phi \circ \hat{\imath}_{n}$ is an embedding of $I$ onto an arc $H \subseteq(I, g)$. According to the previous lemma, for $k$ large enough, $s(n, m+k)$ is one-to-one, so that $s(n, m)=g^{k} \circ s(n, m+k)$ is piecewise monotone with no flat spots and the critical values of $s(n, m)$ are contained in $P(g)$. A similar statement holds for $t(n, m)$.

Now $\hat{\imath}_{m} \circ \pi_{m} \rightarrow$ id uniformly as $m \rightarrow \infty$. Also, $\pi_{m} \circ \hat{\imath}_{m+k}=\pi_{m} \circ \hat{\imath}_{m} \circ f^{k}=$ $f^{k}$, so $t(m, j) \circ s(n, m)=\pi_{j} \circ \Phi^{-1} \circ \hat{\imath}_{m} \circ \pi_{m} \circ \Phi \circ \hat{\imath}_{n} \rightarrow \pi_{j} \circ \hat{\imath}_{n}=f^{n-j}$ uniformly as $m \rightarrow \infty$ and independently of $n$ for fixed $j, 0 \leq j \leq n$. Similarly, $s(m, j) \circ t(n, m) \rightarrow g^{n-j}$.

Claim. For $m$ large enough, each element of $P(g)$ is realized as a critical value of $s(m, 0)$ at least three times.

Choose $H^{\prime}$ to be an arc in $(I, g)$ such that $\pi_{2}\left(H^{\prime}\right)=I$ and $\underline{x}, y \in H^{\prime}$ such that $\pi_{2}(\underline{x})=0, \pi_{2}(\underline{x})=1$. Choose $\varepsilon>0$ such that $g([\varepsilon, 1-\varepsilon])=I$, and $\delta_{1}>$ 0 such that for $\underline{z}, \underline{w} \in(I, g)$, if $|\underline{z}-\underline{w}|<\delta_{1}$, then $\left|\pi_{2}(\underline{z})-\pi_{2}(\underline{w})\right|<\varepsilon$. There is $\delta_{2}>0$ such that if $|\underline{u}-\underline{v}|<\delta_{2}$ for $\underline{u}, \underline{v} \in(I, f)$, then $|\Phi(\underline{u})-\Phi(\underline{v})|<\delta_{1}$. For $m$ large enough, there are $x, y \in I$ such that $\left|\hat{\imath}_{m}(x)-\Phi^{-1}(\underline{x})\right|<\delta_{2} / 2$, $\left|\hat{\imath}_{m}(y)-\Phi^{-1}(y)\right|<\delta_{2} / 2$. For each $i$, there are $x_{i}, y_{i} \in\left[a_{i-1}, a_{i}\right]$ such that $f\left(x_{i}\right)=x, f\left(y_{i}\right)=y$. Finally, since $\hat{\imath}_{m+k} \rightarrow \hat{\imath}_{m} \circ f^{k}$ uniformly as $m \rightarrow \infty, m$ 
can be chosen large enough so that $\left|\hat{\imath}_{m+k}-\hat{\imath}_{m} \circ f^{k}\right|<\delta_{2} / 2$. Then for each $i,\left|\hat{\imath}_{m+1}\left(x_{i}\right)-\Phi^{-1}(\underline{x})\right| \leq\left|\hat{\imath}_{m+1}\left(x_{i}\right)-\hat{\imath}_{m}\left(f\left(x_{i}\right)\right)\right|+\left|\hat{\imath}_{m}\left(f\left(x_{i}\right)\right)-\Phi^{-1}(\underline{x})\right|<$ $\delta_{2} / 2+\delta_{2} / 2=\delta_{2}$. That is, $s(m+1,2)\left(\left[a_{i-1}, a_{i}\right]\right) \supseteq[\varepsilon, 1-\varepsilon]$, so that $s(m+$ $1,1)\left(\left[a_{i-1}, a_{i}\right]\right) \supseteq g[\varepsilon, 1-\varepsilon]=I$. Then, certainly, $s(m+1,0)\left(\left[a_{i-1}, a_{i}\right]\right) \supseteq I$ for each $i$, and since $s(m+1,0)=g \circ s(m+1,1)$, each element of $P(g)$ is realized as a critical value of $s(m+1,0)$ at least three times. The claim is proved.

In the special case under consideration ( $f$ and $g$ have right inverses), take $\alpha_{i}^{-}=\beta_{j}^{-}=0, \alpha_{i}^{+}=\beta_{j}^{+}=1, s_{l, i}=s_{l}$, and $t_{l, j}=t_{l}(1 \leq i \leq n(f)$, $1 \leq j \leq n(g))$ where $s_{l}$ and $t_{l}$ are defined presently.

Let $m$ be large enough so that $\left|s(m+k, 0)-s(m, 0) \circ f^{k}\right|=\mid \pi_{0} \circ \Phi \circ$ $\hat{\imath}_{m+k}-\pi_{0} \circ \Phi \circ \hat{\imath}_{m} \circ f^{k} \mid<\varepsilon_{1} / 2$ for every $k \geq 0$ and so that $s(m, 0)$ satisfies (c). Let $s_{1}=s(m, 0)$. Choose $k$ large enough so that $\mid s(m+k, 0) \circ$ $t(n, m+k)-g^{n} \mid<\varepsilon_{1} / 2$ for $n \geq 0$, and $n$ large enough so that $t(n, m)$ satisfies (c) and $\left|t(n+j, m)-t(n, m) \circ g^{j}\right|<\varepsilon_{2} / 2$ for every $j \geq 0$. Let $t_{1}=t(n, m)$ and $n_{1}=n$. Then $\left|s_{1} \circ t_{1}-g^{n_{1}}\right|=\left|s(m, 0) \circ t(n, m)-g^{n}\right|=$ $\left|s(m, 0) \circ f^{k} \circ t(n, m+k)-g^{n}\right| \leq \mid s(m, 0) \circ f^{k} \circ t(n, m+k)-s(m+k, 0) \circ$ $t(n, m+k)|+| s(m+k, 0) \circ t(n, m+k)-g^{n} \mid<\varepsilon_{1}$.

The inductive definition of $s_{l}$ and $t_{l}$ is now clear.

In the general case, let $L_{i}$ be a subinterval of $I$, maximal with respect to the properties that $f$ is one-to-one on $L_{i}$ and $f\left(L_{i}\right) \supseteq\left[a_{i-1}, a_{i}\right], 0 \leq i \leq n(f)$. Let $\left[\alpha_{i}^{-}, \alpha_{i}^{+}\right]=f\left(L_{i}\right)$ and $f_{i}:\left[\alpha_{i}^{-}, \alpha_{i}^{+}\right] \rightarrow L_{i}$ be the inverse of $\left.f\right|_{L_{i}}$; define $L_{j}^{\prime}, \beta_{j}^{-}, \beta_{j}^{+}, g_{j}$ in a like manner. In the foregoing, replace $f_{1}$ in the definition of $\hat{\imath}_{k}$ by $f_{i}$ to define $\hat{\imath}_{k, i}$ (or $g_{1}$ by $g_{j}$ ) and let $s_{i}(n, m)=\pi_{m} \circ \Phi \circ \hat{\imath}_{n, i}, t_{j}(n, m)=$ $\pi_{m} \circ \Phi \circ \hat{\imath}_{n, j}$. The construction now proceeds as above. That (d) is satisfied is guaranteed by the maximality of $L_{i}$ and $L_{j}^{\prime}$.

Corollary 3.3. With the hypotheses of Proposition 3.2, given a sequence $\left\{\varepsilon_{l}\right\}_{l \in \mathbb{Z}^{+}}$of positive numbers, for $l=1,2, \ldots, 0 \leq i \leq n(f)$ and $0 \leq j \leq n(g)$, there are $n_{l}, m_{l}, s_{l, i}, t_{l, j}$ as above but with (a) improved to

$$
\left|g^{r} \circ s_{l, i} \circ f^{M} \circ t_{p, j}-g^{r+N}\right|<\varepsilon_{l}
$$

and

$$
\left|f^{r^{\prime}} \circ t_{l, j} \circ g^{N^{\prime}} \circ s_{p+1, i}-f^{r^{\prime}+M^{\prime}}\right|<\varepsilon_{l}
$$

where defined, for $p \geq l, 0 \leq r \leq \sum_{i=1}^{l-1} n_{i}, 0 \leq r^{\prime} \leq \sum_{i=1}^{l-1} m_{i}$, and where $M=\sum_{k=l}^{p-1} m_{k}, M^{\prime}=\sum_{k=l}^{p} m_{k}, N=\sum_{k=l}^{p} n_{k}$ and $N^{\prime}=\sum_{k=l+1}^{p} n_{k}$.

TheOREM 3.4. Suppose that $f, g$ are as above with aperiodic transition matrices $A, B$ respectively, and that $(I, f)$ and $(I, g)$ are homeomorphic. Then for $l=1,2, \ldots$, there exist nonnegative matrices $S_{l}, T_{l}$ and positive integers $n_{l}, m_{l}$ such that $S_{l} T_{l}=B^{n_{l}}$ and $T_{l} S_{l+1}=A^{m_{l}}$ : 


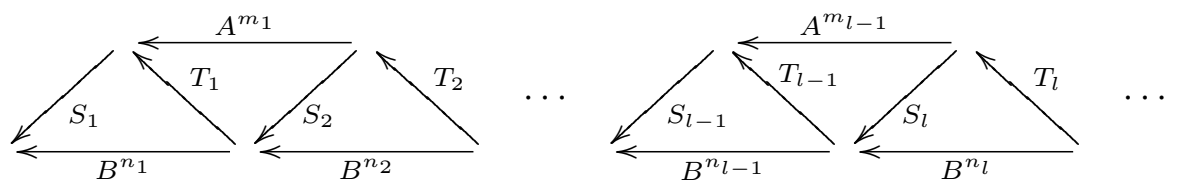

As we mentioned earlier, the proof of Theorem 3.4 is somewhat technical, and is left to $\S 5$. If the maps $s_{l, i}, t_{l, j}$ respected the Markov partitions for $f$ and $g$ in that each $s_{l, i}$ mapped partition elements for $f$ into those for $g$, etc., then the matrices $S_{l}, T_{l}$ could be defined simply as a type of transition matrix for $s_{l, i}, t_{l, j}$. The difficulty arises in showing that the maps $s_{l, i}, t_{l, j}$ are close enough to respecting the partitions for $f$ and $g$ so that what is essentially a transition matrix for each can be defined and has the desired properties.

We are now ready to indicate the relationship between the spectral radii $\alpha$ and $\beta$ of the transition matrices for $f$ and $g$.

Corollary 3.5. Suppose that $(I, f)$ and $(I, g)$ are homeomorphic, where $f, g, A, B, \alpha$ and $\beta$ are as above. Then

(a) there are Perron numbers $c$ and $d$ and positive integers $n$ and $m$ such that $c \alpha=\beta^{m}$ and $d \beta=\alpha^{n}$,

(b) $\mathbb{Q}(\alpha)=\mathbb{Q}(\beta)$.

Proof. (a) We first prove that for each $i$, there are $c_{i}, d_{i}$ such that $S_{i}(\vec{v})=c_{i} \vec{w}$ and $T_{i}(\vec{w})=d_{i} \vec{v}$, where $\vec{v}$ and $\vec{w}$ are right eigenvectors corresponding to the eigenvalues $\alpha$ and $\beta$ respectively. Let $h_{B}$ denote the map on the set $D$ of unit vectors with nonnegative components defined by $h_{B}(\vec{x})=B \vec{x} /|B \vec{x}|$. According to [16, Remark 3.6.9], $h_{B}$ is a contraction on $D$ with fixed point $\vec{w} /|\vec{w}|$. In particular, given any neighbourhood $U$ of $\vec{w} /|\vec{w}|$ in $D$, there is a positive integer $N$ so that if $n \geq N$, then $h_{B}^{n}(D) \subseteq U$. According to Theorem $3.4, \alpha^{k_{l}} S_{1}(\vec{v})=S_{1} A^{k_{l}}(\vec{v})=B^{j_{l}} S_{l}(\vec{v})$, where $k_{l}=\sum_{i=0}^{l} m_{i}$ and $j_{l}=\sum_{i=0}^{l} n_{i}$. Then, normalizing,

$$
\frac{S_{1}(\vec{v})}{\left|S_{1}(\vec{v})\right|}=\frac{B^{j_{l}} S_{l}(\vec{v})}{\left|B^{j_{l}} S_{l}(\vec{v})\right|} .
$$

But $j_{l} \rightarrow \infty$ and $B^{j_{l}} S_{l}(\vec{v}) /\left|B^{j_{l}} S_{l}(\vec{v})\right|$ converges to $\vec{w} /|\vec{w}|$ as $l \rightarrow \infty$. That is, $S_{1}(\vec{v})$ is a scalar multiple of $\vec{w}$. Similarly, $S_{i}(\vec{v})$ is a scalar multiple of $\vec{w}$ for $i>1$ and $T_{i}(\vec{w})$ is a scalar multiple of $\vec{v}$ for $i \geq 1$.

Note that for each $i, c_{i} d_{i+1}$ is an eigenvalue for $S_{i} T_{i+1}$ with eigenvector $\vec{w}$, since $S_{i} T_{i+1}(\vec{w})=S_{i} b_{i+1}(\vec{v})=c_{i} d_{i+1}(\vec{w})$. The matrix $S_{i} T_{i+1}$ is positive and thus has a real and positive eigenvalue which is larger in absolute value than any other and is the only eigenvalue having an associated eigenvector with all components positive ([8, p. 63]). That is, for each $i, c_{i} d_{i+1}$ and $d_{i} c_{i}$ are Perron numbers. 
Now $S_{1} A^{n_{1}} T_{2}=B^{m_{1}+m_{2}}$, and thus

$$
\begin{aligned}
S_{1} A^{n_{1}} T_{2}(\vec{w}) & =B^{m_{1}+m_{2}}(\vec{w}), & S_{1} d_{2} \alpha^{n_{1}}(\vec{v}) & =\beta^{m_{1}+m_{2}}(\vec{w}), \\
S_{1} A^{n_{1}} d_{2}(\vec{v}) & =\beta^{m_{1}+m_{2}}(\vec{w}), & d_{2} \alpha^{n_{1}} c_{1}(\vec{w}) & =\beta^{m_{1}+m_{2}}(\vec{w}),
\end{aligned}
$$

hence $d_{2} \alpha^{n_{1}} c_{1}=\beta^{m_{1}+m_{2}}$. That is, $c \alpha=\beta^{m_{1}+m_{2}}$ for the Perron number $c=d_{2} \alpha^{n_{1}-1} c_{1}$. Similarly, there is a Perron number $d$ and positive integer $n$ such that $d \beta=\alpha^{n}$.

(b) According to (a) and the remarks concluding $\S 2, \alpha \in \mathbb{Q}\left(\beta^{m}\right) \subseteq \mathbb{Q}(\beta)$, and thus $\mathbb{Q}(\alpha) \subseteq \mathbb{Q}(\beta)$. Similarly, $\mathbb{Q}(\beta) \subseteq \mathbb{Q}(\alpha)$, so that $\mathbb{Q}(\alpha)=\mathbb{Q}(\beta)$.

We point out that (a) of Corollary 3.5 is stronger than (b). Recall the definition of $f_{n}$ from $\S 1$, and let $n_{0}, m_{0}$ be positive integers. Since a Perron number is, by definition, an algebraic integer, and a rational algebraic integer is an integer, it follows from (a) that if $\left(I, f_{n_{0}}\right)$ is homeomorphic to $\left(I, f_{m_{0}}\right)$, then $n_{0}$ and $m_{0}$ must have the same prime factors. (This is the result of Watkins mentioned in the introduction.) On the other hand, $\mathbb{Q}(n)=\mathbb{Q}(m)=$ $\mathbb{Q}$ for all positive integers $n, m$.

If $f$ is a transitive Markov map of the interval, then either $f^{2}$ has a dense orbit and $f$ is aperiodic, or there are subintervals $J, K$ of $I$ with $f(J)=K, f(K)=J$ and $|J \cap K|=1$ (cf. [4]). In this second case, $\left.f^{2}\right|_{J}$ and $\left.f^{2}\right|_{K}$ are aperiodic, $\left(I, f^{2}\right)=\left(I,\left.f^{2}\right|_{J}\right) \cup\left(I,\left.f^{2}\right|_{K}\right),\left(I,\left.f^{2}\right|_{J}\right)$ and $\left(I,\left.f^{2}\right|_{K}\right)$ are indecomposable, and $\left|\left(I,\left.f^{2}\right|_{J}\right) \cap\left(I,\left.f^{2}\right|_{K}\right)\right|=1$. If $f, g$ are transitive Markov maps of the interval, and $(I, f)$ and $(I, g)$ are homeomorphic, then either both $f$ and $g$ are aperiodic and 3.5 applies as stated, or there are subintervals $J, K, C, D$ of $I$ such that $\left.f^{2}\right|_{J},\left.f^{2}\right|_{K},\left.g^{2}\right|_{C},\left.g^{2}\right|_{D}$ are aperiodic, $\left(I,\left.f^{2}\right|_{J}\right)$ is homeomorphic to $\left(I,\left.g^{2}\right|_{C}\right),\left(I,\left.f^{2}\right|_{K}\right)$ is homeomorphic to $\left(I,\left.g^{2}\right|_{D}\right)$, and 3.5 applies to the pairs $\left.f^{2}\right|_{J},\left.g^{2}\right|_{C}$ and $\left.f^{2}\right|_{K},\left.g^{2}\right|_{D}$.

4. The period fives. There are three parameter values in $[\sqrt{2}, 2]$ for which the corresponding maps from the tent family have periodic critical point of period five, i.e., periodic kneading sequence of length 5. (We refer the reader to [6] for a description of kneading theory.) We are interested in the inverse limit space of the tent map itself but actually work with the inverse limit space of the associated core map. If $f_{\lambda}$ is in the tent family for $\lambda \in[\sqrt{2}, 2]$, the core map associated with $f_{\lambda}$ is simply $f_{\lambda}$ restricted to the interval $\left[f^{2}(.5), f(.5)\right]$.

The inverse limit space of the core map is an indecomposable continuum; the inverse limit space for the original map is identical to that of the core map except in having an additional composant (of the fixed point $(0,0, \ldots)$ ) entwined with the indecomposable continuum. The inverse limit spaces for the core maps are homeomorphic if and only if so are the inverse limit spaces for the original maps. 
The kneading sequences and corresponding characteristic polynomials of the transition matrices for the core maps are: RLRRC, $P_{1}=x^{4}-x^{3}-x^{2}+$ $x-1$; RLLRC, $P_{2}=x^{4}-x^{3}-x^{2}-x+1$; and RLLLC, $P_{3}=x^{4}-x^{3}-x^{2}-x-1$. Let $\alpha, \lambda$, and $\beta$ denote the parameter values corresponding to RLRRC, RLLRC, and RLLLC respectively. Equivalently, $\alpha, \lambda$, and $\beta$ are the roots of the characteristic polynomials $P_{1}, P_{2}$, and $P_{3}$ that are largest in absolute value and are real and positive. (Each transition matrix for the interval map is irreducible and aperiodic, thus such an eigenvalue for the matrix exists by the Perron-Frobenius Theorem. It is well known that for the tent family parametrized as above, this eigenvalue is the parameter value corresponding to the map, and the entropy of the map is the logarithm of this eigenvalue.) According to Corollary 3.5, to show that corresponding inverse limit spaces are distinct, it is enough to show that so are corresponding algebraic extensions. We first indicate that $\mathbb{Q}(\lambda)$ is distinct from each of $\mathbb{Q}(\alpha)$ and $\mathbb{Q}(\beta)$. Recall that the splitting field of a polynomial $P \in \mathbb{Q}[x]$ is the smallest extension of $\mathbb{Q}$ containing all roots of $P$.

TheOREM 4.1. The extension $\mathbb{Q}(\lambda)$ is distinct from each of $\mathbb{Q}(\alpha)$ and $\mathbb{Q}(\beta)$.

Proof. According to [17, p. 110], if the splitting field of a polynomial contains a root of an irreducible polynomial, then it contains all roots of the polynomial. That is, if $\mathbb{Q}(\lambda)=\mathbb{Q}(\alpha)$, then the splitting field of $P_{1}$ equals the splitting field of $P_{2}$. Let $\lambda_{2}$ denote the second real root of $P_{2}$; then

$$
\lambda, \lambda_{2}=\frac{1}{4}[1+\sqrt{13} \pm \sqrt{2 \sqrt{13}-2}],
$$

while the two complex roots $\lambda_{3}$ and $\lambda_{4}$ of $P_{2}$ are given by

$$
\lambda_{3}, \lambda_{4}=\frac{1}{4}[1+\sqrt{13} \pm \sqrt{-2 \sqrt{13}-2}] .
$$

Then $\mathbb{Q}(\lambda)=\mathbb{Q}\left(\lambda_{2}\right)=\mathbb{Q}(\sqrt{2 \sqrt{13}-2})$, which is of degree 4 , and the splitting field of $P_{2}, \mathbb{Q}\left(\lambda, \lambda_{2}, \lambda_{3}, \lambda_{4}\right)=\mathbb{Q}(\sqrt{2 \sqrt{13}-2}, \sqrt{-2 \sqrt{13}-2})$, which is of degree 2 over $\mathbb{Q}(\sqrt{2 \sqrt{13}-2})$ and thus of degree 8 over $\mathbb{Q}$.

We claim that the degree of $\mathbb{Q}\left(\alpha, \alpha_{2}\right)$ over $\mathbb{Q}$ is 12 , where $\alpha_{2}$ denotes the second real root of $P_{1}$, thus the splitting field of $P_{2}$ cannot equal that of $P_{1}$. Define

$$
y=\frac{1}{3}\left[\sqrt[3]{-\frac{83}{2}-\frac{3}{2} \sqrt{(331)(3)}}+\sqrt[3]{-\frac{83}{2}+\frac{3}{2} \sqrt{(331)(3)}}-1\right] .
$$

According to the CRC Mathematical Tables, the two real roots of $P_{1}$ are of the form $1 / 4+R / 2 \pm D / 2$ where $R^{2}-5 / 4=y$ and $D$ is a term involving 
various radicals we are not concerned with. Then $R \in \mathbb{Q}\left(\alpha, \alpha_{2}\right)$, thus $y \in$ $\mathbb{Q}\left(\alpha, \alpha_{2}\right)$. Since $y$ is of degree 3 ( $y$ is a root of the resolvent cubic equation of $\left.P_{1}, x^{3}+x^{2}+3 x+4=0\right)$, the degree of $\mathbb{Q}\left(\alpha, \alpha_{2}\right)$ over $\mathbb{Q}$ is $3 m$ for some positive integer $m$. But the degree of $\mathbb{Q}\left(\alpha, \alpha_{2}\right)$ over $\mathbb{Q}$ is $4 n$ for some positive integer $n$ since $\alpha$ is of degree 4 . Finally, the degree of $\mathbb{Q}\left(\alpha, \alpha_{2}\right)$ is less than or equal to 16 , since $\alpha_{2}$ is of degree at most 4 , so the degree of $\mathbb{Q}\left(\alpha, \alpha_{2}\right)$ is 12 .

A similar argument applies to show that the degree of $\mathbb{Q}\left(\beta, \beta_{2}\right)$ is 12 .

To prove that $\mathbb{Q}(\alpha)$ and $\mathbb{Q}(\beta)$ are distinct, we note that if $\alpha \in \mathbb{Q}(\beta)$, then $P_{1}$, the irreducible polynomial for $\alpha$, factors over $\mathbb{Q}(\beta)$. One method for indicating that such a factoring does not occur is outlined in van der Waerden [17] and is the basis for the Maple algorithm determining factoring over algebraic extensions. We include only those details relevant to this example.

Suppose that $g(x, \theta)$ is a polynomial with coefficients in $\mathbb{Q}(\theta)$, where $\theta$ has irreducible polynomial $f$ over $\mathbb{Q}$, and that the $n$ conjugates of $\theta$ are given by $\theta=\theta_{1}, \theta_{2}, \ldots, \theta_{n}$. The norm of $g(x, \theta)$ with respect to $\theta$ is

$$
\prod_{i} g\left(x, \theta_{i}\right) .
$$

Since the norm is symmetric in the $\theta_{i}$, the coefficients of the norm are actually in $\mathbb{Q}$. (See $\S 41$ of [17] for a discussion of the norm. One can have Maple calculate the norm as the resultant of $g(x, \theta)$ and $f(\theta)$ with respect to $\theta$.)

The method of van der Waerden involves the norm of $P_{1}(x-y \beta)$ with respect to $\beta$ (i.e., the resultant of $P_{1}(x-y \beta)$ and $P_{3}(\beta)$ with respect to $\beta$ ), which is a polynomial $F(x, y)$ with integer coefficients and of degree 16 in both variables. According to [17, p. 136], if $F(x, y)$ is irreducible over the integers, then $P_{1}$ is irreducible over $\mathbb{Q}(\beta)$. Maple indicates that $F(x, y)$ is irreducible over the integers; we provide a few details. The polynomial $F(x, y)$ does not factor as $f(x) g(y)$, since

$$
F(x, 0)=\left(x^{4}-x^{3}-x^{2}-x+1\right)^{4}
$$

and

$$
\begin{aligned}
F(x, 1)= & x^{16}-8 x^{15}+19 x^{14}+2 x^{13}-63 x^{12}+48 x^{11} \\
& +95 x^{10}-288 x^{9}+640 x^{8}-1042 x^{7}+806 x^{6} \\
& +146 x^{5}-603 x^{4}+214 x^{3}+132 x^{2}-40 x-16
\end{aligned}
$$

are not integer multiples of a common polynomial $f(x)$. Then a nontrivial factoring of $F(x, y)$ must be of the form $f(x, y) g(x, y)$ where the highest power of $x$ appearing in each of $f$ and $g$ is at least 1 , which leads to a nontrivial factoring of $F(x, 1)$ as $f(x, 1) g(x, 1)$. There are a number of de- 
terministic algorithms for factoring polynomials over the integers which vary in efficiency; a simple argument that such a factoring can be performed in a finite number of steps appears in $[17, \S 41]$. According to Maple, $F(x, 1)$ is irreducible over the integers.

We have proved

TheOREM 4.2. Let $f_{\alpha}, f_{\lambda}$ and $f_{\beta}$ be the three maps in the tent family having periodic kneading sequence of length five. The inverse limit spaces $\left(I, f_{\alpha}\right),\left(I, f_{\lambda}\right)$ and $\left(I, f_{\beta}\right)$ are topologically distinct.

We note that Theorem 4.1 can also be proved by pointing out that according to Maple, $P_{2}$ does not factor over $\mathbb{Q}(\alpha)$ or $\mathbb{Q}(\beta)$. We find the argument given more appealing.

The same techniques imply that the inverse limit spaces for any two distinct maps from the tent family having periodic critical point of either period six or seven are topologically distinct. In theory, these techniques might discriminate successfully between nonhomeomorphic inverse limit spaces for maps from the tent family with periodic critical point of arbitrary period. The primary practical limitation is the amount of computer time required to determine whether a factoring of the type described above exists.

5. The diagram of matrices. We now prove Theorem 3.4. Suppose that $f$ and $g$ are as in $\S 3$ with $P(f)=\left\{0=a_{0}<a_{1}<\ldots<a_{n(f)}=1\right\}$, $P(g)=\left\{0=b_{0}<b_{1}<\ldots<b_{n(g)}=1\right\}$, and associated (aperiodic) transition matrices $A$ and $B$ respectively. Also, suppose that $s_{l, i}, t_{l, j}, \varepsilon_{l}, n_{l}, m_{l}$ are as in Corollary 3.3.

To keep notation as simple as possible, as with Proposition 3.2, we first prove each of the results in this section for the case in which the maps $f$ and $g$ have right inverses, and thus single connecting maps $s_{l}$ and $t_{l}$ defined. As we indicate at the end of each proof, the general case follows with very few changes.

LEMMA 5.1. For each $i \in\{0, \ldots, n(f)\}$ and $k \in\{1, \ldots, n(f)\}$ for which $s_{l, k}\left(a_{i}\right)$ is defined, there exists $j \in\{0, \ldots, n(g)\}$ such that $\left|s_{l, k}\left(a_{i}\right)-b_{j}\right|<$ $2 \varepsilon_{l}$. A similar statement holds for $t_{l, j}$.

Proof. Suppose that the maps $f$ and $g$ have right inverses, so that there are maps $s_{l}$ and $t_{l}$ defined as in the proof of 3.2 with $s_{l, i}=s_{l}$ and $t_{l, j}=t_{l}$ for all $i$ and $j$. Fix $i$ and $l$, and suppose that $\left|s_{l}\left(a_{i}\right)-b_{j}\right| \geq 2 \varepsilon_{l}$ for $0 \leq j \leq n(g)$. Since the critical values for $t_{l}$ are precisely the $a_{i}$ 's, there exist $c_{1}<c<c_{2}$ such that $t_{l}(c)=a_{i}, t_{l}\left(c_{1}\right)$ and $t_{l}\left(c_{2}\right)$ are equal to, without loss of generality, $a_{i+1}$, and $t_{l}$ is one-to-one on each of $\left[c_{1}, c\right]$ and $\left[c, c_{2}\right]$. (If $a_{i}$ only occurs as a maximum when it occurs as a critical value for $t_{l}$, then 
replace $a_{i+1}$ with $a_{i-1}$ in what follows.) Suppose that $s_{l}\left(a_{i}\right) \in\left[b_{k}, b_{k+1}\right]$, and, without loss of generality, that for $a$ slightly larger than $a_{i}, s_{l}(a)>s_{l}\left(a_{i}\right)$. Since the critical values for $s_{l}$ are the $b_{k}$ 's and $s_{l}\left(\left[a_{i}, a_{i+1}\right]\right)=I$, there is $a_{i}<d<a_{i+1}$ such that $s_{l}(d)=b_{k+1}$ and $s_{l}$ is one-to-one on $\left[a_{i}, d\right]$. There are $d_{1}$ and $d_{2}$ with $c_{1} \leq d_{1} \leq c \leq d_{2} \leq c_{2}$ and $s_{l} \circ t_{l}\left(d_{i}\right)=b_{k+1}$ for $i=1,2$.

Now $\left|s_{l} \circ t_{l}-g^{n_{l}}\right|<\varepsilon_{l}$, so $g^{n_{l}}\left(d_{1}\right)$ and $g^{n_{l}}\left(d_{2}\right)$ are within $\varepsilon_{l}$ of $b_{k+1}$. On the other hand, $\left|g^{n_{l}}(c)-s_{l}\left(a_{i}\right)\right|<\varepsilon_{l}$, and so $g^{n_{l}}$ has a turning point in $\left(d_{1}, d_{2}\right)$ with associated critical value less than or equal to $b_{k}$, contradicting the fact that $\left|s_{l} \circ t_{l}(x)-b_{k}\right| \geq 2 \varepsilon_{l}$ for all $x \in\left[d_{1}, d_{2}\right]$.

The only possible difficulty in the more general case is that the map $s_{l, k}$ may not be defined on the interval $\left[a_{i}, a_{i+1}\right]$. But in this case, $a_{i}$ occurs as a maximum for each $t_{l, j}$, and the proof can proceed as above with $\left[a_{i-1}, a_{i}\right]$ replacing $\left[a_{i}, a_{i+1}\right]$.

The following technical statement is easily proved.

LEMmA 5.2. Suppose that maps $h, k:\left[c_{1}, c_{2}\right] \rightarrow I$ have the following properties:

(a) $h\left(c_{1}\right)=h\left(c_{2}\right)$,

(b) there is $c \in\left(c_{1}, c_{2}\right)$ such that $h$ is one-to-one on each of $\left[c_{1}, c\right]$ and $\left[c, c_{2}\right]$,

(c) there is $d \in\left[c_{1}, c_{2}\right]$ such that $k$ is one-to-one on $\left[c_{1}, d\right]$ and $\left[d, c_{2}\right]$, and

(d) if $h(c)$ is a maximum, then $k(d)$ is not a maximum; if $h(c)$ is a minimum, then $k(d)$ is not a minimum.

Then $|h-k| \geq \delta / 2$, where $\delta=\left|h(c)-h\left(c_{1}\right)\right|$.

In the following, for $1 \leq i \leq n(f)$ and $1 \leq j \leq n(g)$, let $I_{i}=\left[a_{i-1}, a_{i}\right]$ and $J_{j}=\left[b_{j-1}, b_{i}\right]$. For each $i, j$, define

$$
a_{i}^{\prime}=\frac{a_{i-1}+a_{i}}{2}, \quad b_{j}^{\prime}=\frac{b_{j-1}+b_{j}}{2} .
$$

Suppose that $k, l, j$ are fixed, and let $\left(\left.s_{l, j}\right|_{\left[a_{j-1}, a_{j}\right]}\right)^{-1}\left\{b_{k}^{\prime}\right\}=\left\{x_{1}<x_{2}<\right.$ $\left.\ldots<x_{p}\right\}$. For $1 \leq i \leq p$, let $K\left(x_{i}\right)$ denote the maximal subinterval of $\left[a_{j-1}, a_{j}\right]$ satisfying:

(a) $x_{i} \in K\left(x_{i}\right)$

(b) $\left.s_{l, j}\right|_{K\left(x_{i}\right)}$ is one-to-one, and

(c) $s_{l, j}\left(K\left(x_{i}\right)\right) \subseteq\left[b_{k-1}, b_{k}\right]$.

Lemma 5.3. For sufficiently large $l, 1 \leq i \leq p$, and $0 \leq m \leq n(g)$,

$$
\left(\left[b_{k-1}, b_{k}\right] \backslash s_{l, j}\left(K\left(x_{i}\right)\right)\right) \cap\left(t_{l-1, m}\right)^{-1}\left\{a_{1}^{\prime}, \ldots, a_{n(f)}^{\prime}\right\}=\emptyset .
$$


Proof. Suppose that the maps $f$ and $g$ have right inverses, so that $s_{l, i}=s_{l}$ and $t_{l, j}=t_{l}$ for all $i$ and $j$. Because the critical values of $s_{l}$ are precisely the elements of $P(g), s_{l}$ takes each of $K\left(x_{2}\right), \ldots, K\left(x_{p-1}\right)$ exactly onto $J_{k}$ and (provided $p>1$ ) the right endpoint of $K\left(x_{1}\right)$ and the left endpoint of $K\left(x_{p}\right)$ into $P(g)$. Furthermore, either the left endpoint of $K\left(x_{1}\right)$ is $a_{j-1}$ or $s_{l}$ takes this endpoint into $P(g)$ and the right endpoint of $K\left(x_{p}\right)$ is either $a_{j}$ or is taken into $P(g)$ by $s_{l}$. That is, if $s_{l}$ does not take $K\left(x_{1}\right)$ onto $J_{k}$, then $K\left(x_{1}\right)=\left[a_{j-1}, a\right]$ where either $s_{l}(a) \in P(g)$ or $a=a_{j}$. In the latter case, $p=1$ and $K\left(x_{1}\right)=\left[a_{j-1}, a_{j}\right]$, so that $s_{l}\left(K\left(x_{1}\right)\right)=$ $s_{l}\left(\left[a_{j-1}, a_{j}\right]\right)=I$ and the result holds. If $s_{l}\left(K\left(x_{1}\right)\right) \neq J_{k}$, then suppose without loss of generality that $s_{l}$ is orientation preserving on $K\left(x_{1}\right)$, i.e., that $b_{k-1}<s_{l}\left(a_{j-1}\right)<b_{k}^{\prime}$.

Choose $c \in I \backslash\{0,1\}$ such that $t_{l}(c)=a_{j-1}$ and $c$ is a critical point of $t_{l}$.

Claim. There are $c_{1}<c<c_{2}$ such that $s_{l} \circ t_{l}$ is one-to-one on each of $\left[c_{1}, c\right]$ and $\left[c, c_{2}\right]$ and either $s_{l} \circ t_{l}\left(c_{1}\right)=b_{k-1}=s_{l} \circ t_{l}\left(c_{2}\right)$ or $s_{l} \circ t_{l}\left(c_{1}\right)=$ $b_{k}=s_{l} \circ t_{l}\left(c_{2}\right)$.

Proof of claim. Suppose that $a_{j-1}=0$. Then there are $e_{1}<c<e_{2}$ such that $t_{l}$ is one-to-one on $\left[e_{1}, c\right]$ and $\left[c, e_{2}\right]$ and $t_{l}\left(e_{i}\right)=a_{j}$ for $i=1,2$, and thus $c_{1}, c_{2}$ such that $e_{1}<c_{1}<c<c_{2}<e_{2}, t_{l}$ is one-to-one on $\left[c_{1}, c\right]$ and $\left[c, c_{2}\right]$ and $t_{l}\left(c_{i}\right)=a$.

Suppose that $a_{j-1} \neq 0$. Then there is $b<a_{j-1}$ such that $s_{l}(b)=b_{k-1}$ and $s_{l}$ is one-to-one on $\left[b, a_{j-1}\right]$. There are $e_{1}<c<e_{2}$ such that $t_{l}$ is oneto-one on $\left[e_{1}, c\right]$ and $\left[c, e_{2}\right]$ and either $t_{l}\left(e_{i}\right)=a_{j}$ for $i=1,2$ or $t_{l}\left(e_{i}\right)=a_{j-2}$ for $i=1,2$. Then there are $c_{1}, c_{2}$ such that $e_{1}<c_{1}<c<c_{2}<e_{2}, t_{l}$ is one-to-one on $\left[c_{1}, c\right]$ and $\left[c, c_{2}\right]$ and either $t_{l}\left(c_{i}\right)=a$ or $t_{l}\left(c_{i}\right)=b$. The claim is proved.

We consider the two cases separately. We assume without loss of generality that $\left\{\varepsilon_{l}\right\}_{l \in \mathbb{Z}^{+}}$is a decreasing sequence converging to 0 , and choose $l$ large enough so that $2 \varepsilon_{l}<\min _{b \in P(g)}\left\{d\left(b, g^{-1}(P(g)) \backslash\{b\}\right)\right\}$.

Case I: $s_{l} \circ t_{l}\left(c_{1}\right)=b_{k}=s_{l} \circ t_{l}\left(c_{2}\right)$. Assume that $2 \varepsilon_{l}<b_{k}-b_{k}^{\prime}=$ $\left(b_{k}-b_{k-1}\right) / 2$. If $\left.g^{n_{l}}\right|_{\left[c_{1}, c_{2}\right]}$ were one-to-one, then according to Lemma 5.2, $\left|g^{n_{l}}-s_{l} \circ t_{l}\right| \geq\left(b_{k}-b_{k-1}\right) / 4 \geq \varepsilon_{l}$ on $\left[c_{1}, c_{2}\right]$. Then $g^{n_{l}}$ has a turning point in $\left[c_{1}, c_{2}\right]$, so either $g^{n_{l}}(d)=b_{k-1}$ for some $d \in\left[c_{1}, c_{2}\right]$, or $g^{n_{l}}(d)=b_{k}$ for some $d$. If the former case does not occur, then $g^{n_{l}}$ must have a maximum at $d$ and be one-to-one on $\left[c_{1}, d\right]$ and $\left[d, c_{2}\right]$. But then Lemma 5.2 implies that $\left|g^{n_{l}}-s_{l} \circ t_{l}\right| \geq \varepsilon_{l}$. Thus there exists $d \in\left[c_{1}, c_{2}\right]$ such that $g^{n_{l}}(d)=$ $b_{k-1}$.

Since $\varepsilon_{l}<\min _{b \in P(g)}\left\{d\left(b, g^{-1}(P(g)) \backslash\{b\}\right)\right\}$, the map $g$ restricted to $\left[b_{k-1}, s_{l} \circ t_{l}(d)\right]=\left[g^{n_{l}}(d), s_{l} \circ t_{l}(d)\right]$ is one-to-one. Thus diam $\left(g\left[b_{k-1}, s_{l}\left(a_{j-1}\right)\right]\right)$ $\leq \operatorname{diam}\left(g\left[b_{k-1}, s_{l} \circ t_{l}(d)\right]\right)=\left|g \circ s_{l} \circ t_{l}(d)-g\left(b_{k-1}\right)\right|<\varepsilon_{l}$ (where the last in- 
equality follows from Corollary 3.3). But then $\left.g\right|_{g\left[b_{k-1}, s_{l} \circ t_{l}(d)\right]}$ is one-to-one. Continuing, $\operatorname{diam}\left(g^{r}\left[b_{k-1}, s_{l}\left(a_{j-1}\right)\right]\right) \leq \operatorname{diam}\left(g^{r}\left[b_{k-1}, s_{l} \circ t_{l}(d)\right]\right)<\varepsilon_{l}$ for $0 \leq r \leq \sum_{i=1}^{l-1} n_{i}$.

Case II: $s_{l} \circ t_{l}\left(c_{1}\right)=b_{k-1}=s_{l} \circ t_{l}\left(c_{2}\right)$. In this case, $g^{n_{l}}$ can have at most one critical point in $\left[c_{1}, c_{2}\right]$, and if there is a critical value, it must be $b_{k-1}$. Suppose then that there exists $d \in\left(c_{1}, c_{2}\right)$ such that $g^{n_{l}}(d)=b_{k-1}$. Then $g^{n_{l}}$ is one-to-one on $\left[c_{1}, d\right]$ and on $\left[d, c_{2}\right]$.

If $g^{n_{l}}$ has a minimum at $d$, then since $\left|b_{k}-s_{l}\left(a_{j-1}\right)\right|<\varepsilon_{l}, g$ is one-to-one on $\left[b_{k-1}, s_{l}\left(a_{j-1}\right)\right]$ and $g \circ s_{l} \circ t_{l}$ and $g^{n_{l}+1}$ satisfy the hypotheses of Lemma 5.2. Then $\left|g \circ s_{l} \circ t_{l}-g^{n_{l}+1}\right|<\varepsilon_{l}$ implies that $\left|g\left(b_{k-1}\right)-g \circ s_{l}\left(a_{j-1}\right)\right|<2 \varepsilon_{l}$, thus $g$ is one-to-one on $g\left[b_{k-1}, s_{l}\left(a_{j-1}\right)\right]$. Continuing, $\operatorname{diam}\left(g^{r}\left[b_{k-1}, s_{l}\left(a_{j-1}\right)\right]\right)<$ $2 \varepsilon_{l}$ for $0 \leq r \leq \sum_{i=1}^{l-1} n_{i}$.

If $g^{n_{l}}$ has a maximum at $d$ or does not turn at $d$, then $b_{k-1}$ is not an endpoint of $I$ and $g$ turns at $b_{k-1}$. Since the map $g$ is one-to-one on $\left[b_{k-1}-2 \varepsilon_{l}, b_{k-1}\right]$ and $\left[b_{k-1}, b_{k-1}+2 \varepsilon_{l}\right]$, it follows that $g \circ s_{l} \circ t_{l}$ has a single maximum (or minimum) in $\left[c_{1}, c_{2}\right]$ while $g \circ g^{n_{l}}$ has a single minimum (or maximum) in $\left[c_{1}, c_{2}\right]$. By Lemma 5.2, $g^{r} \circ s_{l} \circ t_{l}\left[c_{1}, c_{2}\right]$, and hence $g^{r}\left[b_{k-1}, s_{l}\left(a_{j-1}\right)\right]$, remains small.

If there is no $d \in\left(c_{1}, c_{2}\right)$ for which $g^{n_{l}}(d)=b_{k-1}$, then $g^{n_{l}}$ and $g^{n_{l}+1}$ are one-to-one on $\left[c_{1}, c_{2}\right]$, and arguments similar to the above apply.

We have proved that in either case, $g^{r}\left[b_{k-1}, s_{l}\left(a_{j-1}\right)\right]$ remains small for $0 \leq r \leq \sum_{i=1}^{l-1} n_{i}$. Now suppose that $t_{l-1}\left[b_{k-1}, s_{l}\left(a_{j-1}\right)\right] \cap\left\{a_{1}^{\prime}, \ldots, a_{n(f)}^{\prime}\right\} \neq \emptyset$, say $a_{i}^{\prime} \in t_{l-1}\left[b_{k-1}, s_{l}\left(a_{j-1}\right)\right]$. Also, $t_{l-1}\left(s_{l}\left(a_{j-1}\right)\right) \in t_{l-1}\left[b_{k-1}, s_{l}\left(a_{j-1}\right)\right]$ and, if $l$ is large enough, $t_{l-1}\left(s_{l}\left(a_{j-1}\right)\right) \approx f^{m_{l-1}}\left(a_{j-1}\right) \in P(f)$. According to Lemma 2.1, there is $N$ such that for all $n \geq N, f^{n}\left(t_{l-1}\left[b_{k-1}, s_{l}\left(a_{j-1}\right)\right]\right)=I$. Let $l$ and $i^{\prime}$ be large enough so that $n=\sum_{i=i^{\prime}}^{l-1} m_{i} \geq N$ and $\varepsilon_{i^{\prime}}<1 / 4$. Let $r=\sum_{i=i^{\prime}}^{l-1} n_{i}$. Then $\left|s_{i^{\prime}} \circ f^{n} \circ t_{l-1}-g^{r}\right|<1 / 4$ (Corollary 3.3), while $s_{i^{\prime}} \circ f^{n} \circ$ $t_{l-1}\left[b_{k-1}, s_{l}\left(a_{j-1}\right)\right]=I$ and $g^{r}\left[b_{k-1}, s_{l}\left(a_{j-1}\right)\right]$ is small. This contradiction proves the lemma in the simpler case.

In the more general case, $s_{l, j}$ may not be defined for $x<a_{j}$. In this case, since $a_{j}=\alpha_{j}^{-}, a_{j}$ occurs as a minimum for each $t_{l, k}$, and hence $c$ can be chosen so that Case I holds. The proof then follows.

We are now ready to complete the proof of Theorem 3.4. For each $l$, the matrices $S_{l}$ and $T_{l}$ that appear in the statement of Theorem 3.4 are defined by

$$
\left(S_{l}\right)_{k j}=\#\left(\left.s_{l, j}\right|_{\left[a_{j-1}, a_{j}\right]}\right)^{-1}\left(b_{k}^{\prime}\right), \quad\left(T_{l}\right)_{i k}=\#\left(\left.t_{l, k}\right|_{\left[b_{k-1}, b_{k}\right]}\right)^{-1}\left(a_{i}^{\prime}\right) .
$$

Proof of Theorem 3.4. The proof of the general case is identical to that for the simpler case, in which $s_{l, i}=s_{l}$ and $t_{l, j}=t_{l}$ for all $i$ and $j$. We avoid the more complicated notation of the general case and merely 
present the argument for the simpler case. Fix $k \in\{1, \ldots, n(g)\}$ and let $K\left(x_{1}\right), \ldots, K\left(x_{p}\right) \subseteq I_{j}$ be as above. Let $\left\{y_{1}, \ldots, y_{r}\right\}=\left[\left(t_{l-1}\right)^{-1}\left(a_{i}^{\prime}\right)\right] \cap$ $J_{k}$. Then $r=\left(T_{l-1}\right)_{i k}, s_{l}\left(K\left(x_{n}\right)\right) \supseteq\left\{y_{1}, \ldots, y_{r}\right\}$ for $1 \leq n \leq p$ and $l$ large enough by Lemma 5.3 , and $p=\left(S_{l}\right)_{k j}$. Since $\left.s_{l}\right|_{K\left(x_{n}\right)}$ is one-toone, $\#\left(\left.s_{l}\right|_{I_{j}}\right)^{-1}\left(\left\{y_{1}, \ldots, y_{r}\right\}\right)=r p=\left(T_{l-1}\right)_{i k}\left(S_{l}\right)_{k j}$. According to Lemma 5.1 , for $l$ large enough, each point of $\left(t_{l-1}\right)^{-1}\left(\left\{a_{1}^{\prime}, \ldots, a_{n(f)}^{\prime}\right\}\right)$ is in exactly one of the $J_{k}$ 's, hence $\#\left(\left.t_{l-1} \circ s_{l}\right|_{I_{j}}\right)^{-1}\left(a_{i}^{\prime}\right)=\sum_{k=1}^{n(g)}\left(T_{l-1}\right)_{i k}\left(S_{l}\right)_{k j}=$ $\left(T_{l-1} S_{l}\right)_{i j}$.

Now we argue that $\#\left(\left.t_{l-1} \circ s_{l}\right|_{I_{j}}\right)^{-1}\left(a_{i}^{\prime}\right)=\#\left(\left.f^{m_{l-1}}\right|_{I_{j}}\right)^{-1}\left(a_{i}^{\prime}\right)$. Let $L_{1}, \ldots, L_{q}$ be the distinct subintervals of $I_{j}$ with $f^{m_{l-1}}\left(L_{n}\right)=I_{i},\left.f^{m_{l-1}}\right|_{L_{n}}$ one-to-one $(1 \leq n \leq q)$ and $q=\left(A^{m_{l-1}}\right)_{i j}$. Since $\left|t_{l-1} \circ s_{l}-f^{m_{l-1}}\right|<$ $\varepsilon_{l-1}, a_{i}^{\prime} \in t_{l-1} \circ s_{l}\left(L_{n}\right)$ for each $n$ as long as $l$ is sufficiently large. Thus $\#\left(\left.t_{l-1} \circ s_{l}\right|_{I_{g}}\right)^{-1}\left(a_{i}^{\prime}\right) \geq \#\left(\left.f^{m_{l-1}}\right|_{I_{j}}\right)^{-1}\left(a_{i}^{\prime}\right)$.

Suppose that \# $\left(\left.t_{l-1} \circ s_{l}\right|_{L_{n}}\right)^{-1}\left(a_{i}^{\prime}\right) \geq 2$. The critical values of $s_{l}$ lie in $P(g)$, and those of $t_{l-1}$ lie in $P(f)$, so the critical values of $t_{l-1} \circ s_{l}$ lie close to $t_{l-1}(P(g)) \cup P(f)$ (Lemma 5.1). That is, the critical values of $t_{l-1} \circ s_{l}$ lie far away from $a_{i}^{\prime}$. Then there are elements $c_{1}<c<c_{2}$ of $L_{n}$ with $t_{l-1} \circ s_{l}\left(c_{1}\right)=t_{l-1} \circ s_{l}\left(c_{2}\right)=a_{i}^{\prime}$ and $\left.\left(t_{l-1} \circ s_{l}\right)\right|_{\left[c_{1}, c\right]}$ and $\left.\left(t_{l-1} \circ s_{l}\right)\right|_{\left[c, c_{2}\right]}$ one-to-one. Since $f^{m_{l-1}}$ is one-to-one on $\left[c_{1}, c_{2}\right]$, Lemma 5.2 implies that $\operatorname{diam}\left(t_{l-1} \circ s_{l}\left(\left[c_{1}, c\right]\right)\right) \leq 2 \varepsilon_{l}$. But $t_{l-1} \circ s_{l}\left(c_{1}\right)=a_{i}^{\prime}$ and $t_{l-1} \circ s_{l}(c)$ is close to $t_{l-1}(P(g)) \cup P(f)$, so this is a contradiction.

Finally, since the result holds for large enough $l$, it can be assumed to hold for all $l$ by considering a tail of the original sequence.

\section{References}

[1] J. M. Aarts and R. J. Fokkink, The classification of solenoids, Proc. Amer. Math. Soc. 111 (1991), 1161-1163.

[2] M. Barge, Horseshoe maps and inverse limits, Pacific J. Math. 121 (1986), 29-39.

[3] M. Barge and S. Holte, Nearly one-dimensional Henon attractors and inverse limits, preprint.

[4] M. Barge and J. Martin, Chaos, periodicity and snake-like continua, Trans. Amer. Math. Soc. 289 (1985), 355-365.

[5] R. H. Bing, A simple closed curve is the only homogeneous bounded plane continuum that contains an arc, Canad. J. Math. 12 (1960), 209-230.

[6] P. Collet and J. P. Eckmann, Iterated Maps on the Interval as Dynamical Systems, Birkhäuser, Boston, 1980.

[7] W. Dębski, On topological types of the simplest indecomposable continua, Colloq. Math. 49 (1985), 203-211.

[8] F. R. Gantmacher, The Theory of Matrices, Vol. II, Chelsea, New York, 1959.

[9] J. Guckenheimer, Sensitive dependence to initial conditions for one-dimensional maps, Comm. Math. Phys. 70 (1979), 133-160.

[10] S. Holte, Generalized horseshoe maps and inverse limits, Pacific J. Math. 156 (1992), 297-305. 
[11] S. Holte, Inverse limits of Markov interval maps, preprint.

[12] S. Holte and R. Roe, Inverse limits associated with the forced van der Pol equation, preprint.

[13] D. A. Lind, The entropies of topological Markov shifts and a related class of algebraic integers, Ergodic Theory Dynamical Systems 4 (1984), 283-300.

[14] M. C. McCord, Inverse limit sequences with covering maps, Trans. Amer. Math. Soc. 114 (1965), 197-209.

[15] J. Mioduszewski, Mappings of inverse limits, Colloq. Math. 10 (1963), 39-44.

[16] C. Robinson, Introduction to the Theory of Dynamical Systems, manuscript, July 1993.

[17] B. L. van der Waerden, Modern Algebra, Ungar, New York, 1953.

[18] W. T. Watkins, Homeomorphic classification of certain inverse limit spaces with open bonding maps, Pacific J. Math. 103 (1982), 589-601.

[19] R. Williams, One-dimensional nonwandering sets, Topology 6 (1967), 473-487.

DEPARTMENT OF MATHEMATICAL SCIENCES

MONTANA STATE UNIVERSITY

BOZEMAN, MONTANA 59717

U.S.A.

E-mail: BARGE@MATH.MONTANA.EDU
DEPARTMENT OF MATHEMATICS

UNIVERSITY OF CHARLESTON CHARLESTON, SOUTH CAROLINA 29424

U.S.A.

E-mail: DIAMONDB@COFC.EDU

Received 3 November 1993; in revised form 8 May 1994 